\title{
Common Fixed Point Theorems in Metric Space by Altering Distance Function
}

\author{
Vishnu Narayan Mishra1,2*, Balaji Raghunath Wadkar ${ }^{3}$, Ramakant Bhardwaj ${ }^{4}$, Idrees A. Khan ${ }^{5}$, \\ Basant Singh ${ }^{3}$
}

\author{
${ }^{1}$ Applied Mathematics \& Humanities Department, S.V. National Institute of Technology, Gujarat, India \\ ${ }^{2}$ Department of Mathematics, Indira Gandhi National Tribal University, Madhya Pradesh, India \\ ${ }^{3}$ Department of Mathematics, AISECT University, Bhopal, India \\ ${ }^{4}$ Department of Mathematics, TIT Group of Institutes, Anand Nagar, India \\ ${ }^{5}$ Department of Mathematics, Integral University, Lucknow, India \\ Email: vishnunarayanmishra@gmail.com,wadkar.bhardwaj@gmail.com,rkbhardwaj100@gmail.com, \\ idrees_maths@yahoo.com,wbrlatur@gmail.com
}

How to cite this paper: Mishra, V.N. Wadkar, B.R., Bhardwaj, R., Khan, I.A. and Singh, B. (2017) Common Fixed Point Theorems in Metric Space by Altering Distance Function. Advances in Pure Mathematics, 7, 335-344.

https://doi.org/10.4236/apm.2017.76020

Received: April 4, 2017

Accepted: June 6, 2017

Published: June 9, 2017

Copyright $\odot 2017$ by authors and Scientific Research Publishing Inc. This work is licensed under the Creative Commons Attribution International License (CC BY 4.0).

http://creativecommons.org/licenses/by/4.0/

\begin{abstract}
In the present paper, we prove two theorems. In first theorem, we prove fixed point result for self-maps in the metric space under contractive condition of integral type by altering distance. In second result, we prove a unique common fixed point theorem by considering four sub compatible maps under a contractive condition of integral type.
\end{abstract}

\section{Keywords}

Altering Distance Function, Sub Compatible

\section{Introduction and preliminaries}

\section{Open Access}

in metric space. Aliouche [2] proved common fixed point results in symmetric space for weakly compatible mappings under contractive condition of integral type. In [3], Babu generalized and proved fixed point results using control function. Later Bouhadjera and Godet [4] generalized concept of pair sub compatible maps and proved fixed point results. Also Chaudhari [5] [6], Chugh \& Kumar [7], Naidu [8], Sastry et al. [9] generalized and proved some fixed point results. Recently in [10] [11], Hosseni used contractive rule of integral type by altering distance and generalized common fixed point results. Many authors proved fixed point results with different techniques in different spaces (see [12]-[17]). In [18] [19] [20] [21], Wadkar et al. proved fixed point theorems using the concept of soft metric space. In the present paper, we prove two theorems on fixed point 
under contraction rule of integral type in metric space by altering distance function, first for self map and second for a pair of sub compatible maps. Our results are motivated by V. R. Hosseni, Neda Hosseni.

Definition 1.1: A function $\psi: R^{+} \rightarrow R^{+}=[0,1)$ is an altering distance functions if $\psi$ is continuous with monotone increasing in all variables and $\psi\left(x_{1}, x_{2}, x_{3}, \cdots, x_{n}\right)=0$ if $x_{1}=x_{2}=x_{3}=\cdots=x_{n}=0$.

The collection of all altering distance is denoted by $\Psi_{n}$.

Now let us define a function $m(y)$ by $m(y)=\psi(y, y, y, y, \cdots, y)$ for $y \in[0, \infty)$, clearly $m(y)=0$ if and only if $y=0$.

Examples of $\psi$ are $\psi\left(f_{1}, f_{2}, f_{3}, \cdots, f_{n}\right)=\mu \max \left\{f_{1}, f_{2}, f_{3}, \cdots, f_{n}\right\}$, for $\mu>0$,

$$
\psi\left(f_{1}, f_{2}, f_{3}, \cdots, f_{n}\right)=f_{n}^{a_{1}}+f_{n}^{a_{2}}+f_{n}^{a_{3}}+\cdots+f_{n}^{a_{n}}, a_{1}, a_{2}, \cdots, a_{n} \geq 1 .
$$

Definition 1.2: The maps $p, q: E \rightarrow E$ of metric space $(E, \sigma)$ are called as sub compatible if and only if the sequence $\left\{e_{n}\right\}$ in $E$ such that $\lim _{n \rightarrow \infty} p_{n}=\lim _{n \rightarrow \infty} q_{n}=t, t \in E$ and which satisfies $\lim _{n \rightarrow \infty} \sigma\left(p q e_{n}, q p e_{n}\right)=0$.

Example 1.3: Let $E=[0, \infty)$ we define $p \& \stackrel{n \rightarrow \infty}{q}$ with metric $\sigma$ as follows

$$
p(e)=e^{2} \quad \& \quad q(e)= \begin{cases}e+6 & \text { if } e \in[4,9] \cup(27, \infty) \\ e^{2}, & \text { if } e \in[9,27]\end{cases}
$$

Let us define the sequence $\left\{e_{n}\right\}$ in $E$ as $e_{n}=3+\frac{1}{n}$, for $n=0,1,2, \cdots$ then

$$
\lim _{n \rightarrow \infty} p e_{n}=\lim _{n \rightarrow \infty} e_{n}^{2}=9=\lim _{n \rightarrow \infty} q e_{n}=\lim _{n \rightarrow \infty}\left(e_{n}+6\right),
$$

and $\lim _{n \rightarrow \infty} p q\left(e_{n}\right)=\lim _{n \rightarrow \infty} p\left(e_{n}+6\right)=\lim _{n \rightarrow \infty}\left(e_{n}+6\right)^{2}=81$ when $n \rightarrow \infty$,

$$
\lim _{n \rightarrow \infty} \text { qpe }_{n}=\lim _{n \rightarrow \infty} q e_{n}^{2}=\left(e_{n}^{2}\right)^{2}=\lim _{n \rightarrow \infty}\left(e_{n}\right)^{4}=81 \text { when } n \rightarrow \infty \text {. }
$$

Thus, we have $\lim _{n \rightarrow \infty} \sigma\left(p q e_{n}, q p e_{n}\right)=0$.

Hence maps $p$ and $q$ are sub-compatible.

On the other hand, we have $p e=q e$ if and only if $e=3$, $p q(3)=p(9)=81$ and $q p(3)=q(9)=9+6=15$.

Then $p(3)=9=q(3)$ but $p q(3)=81 \neq 15=q p(3)$, hence $p$ and $q$ are not OWC (Oscillatory weakly commuting).

\section{Main Result}

Theorem 2.1: Let us consider the mappings $U, V: E \rightarrow E$ of complete metric space $(E, \sigma)$ be such that for all $c, d \in E$

$$
\begin{aligned}
\int_{0}^{\phi_{1}(\sigma(U c, V d))} \eta(y) \mathrm{d} y \leq & \int_{0}^{\psi_{1}\left(\sigma(c, d), \sigma(U c, c), \sigma(V d, d), \frac{1}{2}\{\sigma(V d, c)+\sigma(U c, d)\}, \frac{1}{2}, \frac{1}{2}(\sigma(c, d)+\sigma(U c, c)\}\right)} \eta(y) \mathrm{d} y \\
& -\int_{0}^{\psi_{2}\left(\sigma(c, d), \sigma(U c, c), \sigma(V d, d), \frac{1}{2}\{\sigma(V d, c)+\sigma(U c, d)), \frac{1}{2}\left\{\frac{1}{2}\{(c, d)+\sigma(U c, c)\}\right)\right.} \eta(y) \mathrm{d} y,
\end{aligned}
$$

where $\psi_{1}, \psi_{2} \in \Psi_{5}$ with $\phi_{1}=\psi(e, e, e, e, e), \quad e \in[0, \infty)$ and Lebesgue-integrable mapping $\eta: R^{+} \rightarrow R^{+}$, which is positive, sum able, and for each $\in>0, \int_{0}^{\epsilon} \eta(y) \mathrm{d} y>0$, then there exist a unique common fixed point in $E$ for $U$ 
and $V$.

Proof: Consider arbitrary point $e_{0}$ of $E$, for $n=1,2,3, \cdots$ we have

$$
\begin{gathered}
e_{2 n+1}=U e_{2 n} \\
\text { and } e_{2 n+2}=V e_{2 n+1} .
\end{gathered}
$$

Let $r_{n}=\sigma\left(e_{n}, e_{n+1}\right)$

Substituting $c=e_{2 n}$ and $d=e_{2 n+1}$ in Equation (8), then for all $n=1,2,3, \cdots$ we have

$$
\begin{aligned}
& \int_{0}^{\phi_{1}\left(\sigma\left(U e_{2 n}, V e_{2 n+1}\right)\right)} \eta(y) \mathrm{d} y \leq \int_{0}^{\phi_{1}\left(\sigma\left(e_{2 n+1}, e_{2 n+2}\right)\right)} \eta(y) \mathrm{d} y \\
& \leq \int_{0}^{\psi_{1}\left(\sigma\left(e_{2 n}, e_{2 n+1}\right), \sigma\left(U e_{2 n}, e_{2 n}\right), \sigma\left(V e_{2 n+1}, e_{2 n+1}\right), \frac{1}{2}\left\{\sigma\left(V e_{2 n+1}, e_{2 n}\right)+\sigma\left(U e_{2 n}, e_{2 n+1}\right)\right\}, \frac{1}{2}\left\{\sigma\left(e_{2 n}, e_{2 n+1}\right)+\sigma\left(U e_{2 n}, e_{2 n}\right)\right\}\right)} \eta(y) \mathrm{d} y \\
& \quad-\int_{0}^{\psi_{2}}\left(\sigma\left(e_{2 n}, e_{2 n+1}\right), \sigma\left(U e_{2 n}, e_{2 n}\right), \sigma\left(V e_{2 n+1}, e_{2 n+1}\right), \frac{1}{2}\left\{\sigma\left(V e_{2 n+1}, e_{2 n}\right)+\sigma\left(U e_{2 n}, e_{2 n+1}\right)\right\}, \frac{1}{2}\left\{\sigma\left(e_{2 n}, e_{2 n+1}\right)+\sigma\left(U e_{2 n}, e_{2 n}\right)\right\}\right) \eta(y) \mathrm{d} y \\
& \leq \int_{0}^{\psi_{1}\left(\sigma\left(e_{2 n}, e_{2 n+1}\right), \sigma\left(e_{2 n+1}, e_{2 n}\right), \sigma\left(e_{2 n+2}, e_{2 n+1}\right), \frac{1}{2}\left\{\sigma\left(e_{2 n+2}, e_{2 n}\right)+\sigma\left(e_{2 n+1}, e_{2 n+1}\right)\right\}, \frac{1}{2}\left\{\sigma\left(e_{2 n}, e_{2 n+1}\right)+\sigma\left(e_{2 n+1}, e_{2 n}\right)\right\}\right)} \eta(y) \mathrm{d} y \\
& \quad-\int_{0}^{\psi_{2}\left(\sigma\left(e_{2 n}, e_{2 n+1}\right), \sigma\left(e_{2 n+1}, e_{2 n}\right), \sigma\left(e_{2 n+2}, e_{2 n+1}\right), \frac{1}{2}\left\{\sigma\left(e_{2 n+2}, e_{2 n}\right)+\sigma\left(e_{2 n+1}, e_{2 n+1}\right)\right\}, \frac{1}{2}\left\{\sigma\left(e_{2 n}, e_{2 n+1}\right)+\sigma\left(e_{2 n+1}, e_{2 n}\right)\right\}\right)} \eta(y) \mathrm{d} y
\end{aligned}
$$

Using Equation (9) for all $n=1,2,3, \cdots$ we get

$$
\begin{aligned}
\int_{0}^{\phi_{1}\left(r_{2 n+1}\right)} \eta(y) \mathrm{d} y \leq & \int_{0}^{\psi_{1}\left(r_{2 n}, r_{2 n}, r_{2 n+1} \frac{1}{2}\left\{r_{2 n+1}+r_{2 n}+0\right\}, \frac{1}{2}\left\{r_{2 n}+r_{2 n}\right\}\right)} \eta(y) \mathrm{d} y \\
& -\int_{0}^{\psi_{2}\left(r_{2 n}, r_{2 n}, r_{2 n+1} \frac{1}{2}\left\{r_{2 n+1}+r_{2 n}+0\right\}, \frac{1}{2}\left\{r_{2 n}+r_{2 n}\right\}\right)} \eta(y) \mathrm{d} y
\end{aligned}
$$

As $r_{2 n+1}>r_{2 n}$ implies that $r_{2 n+1}+r_{2 n} \leq 2 r_{2 n+1}$, so we have

$$
\int_{0}^{\phi_{1}\left(r_{2 n+1}\right)} \eta(y) \mathrm{d} y \leq \int_{0}^{\psi_{1}\left(r_{2 n}, r_{2 n}, r_{2 n+1} r_{2 n+1}, r_{2 n}\right)} \eta(y) \mathrm{d} y=\int_{0}^{\phi_{1}\left(r_{2 n+1}\right)} \eta(y) \mathrm{d} y
$$

Now by monotone increase of $\psi_{1}$ in all variables and using the property that $\psi_{2}\left(r_{2 n}, r_{2 n}, r_{2 n+1}, r_{2 n+1}, r_{2 n}\right) \neq 0$ whenever $r_{2 n+1} \neq 0$, we get a contradiction i.e. $r_{2 n+1}$ not greater than $r_{2 n}$. Hence we have $r_{2 n+1} \leq r_{2 n}$, for

$$
n=0,1,2,3, \cdots
$$

Substituting $c=e_{2 n-1}, d=e_{2 n}$ in Equation (8) we have

$$
\int_{0}^{\phi_{1}\left(r_{2 n}\right)} \eta(y) \mathrm{d} y \leq \int_{0}^{\psi_{1}\left(r_{2 n-1}, r_{2 n-1}, r_{2 n}, r_{2 n}, r_{2 n-1}\right)} \eta(y) \mathrm{d} y-\int_{0}^{\psi_{2}\left(r_{2 n-1}, r_{2 n-1}, r_{2 n}, r_{2 n}, r_{2 n-1}\right)} \eta(y) \mathrm{d} y
$$

By using (12) we consider

$$
r_{2 n+2} \leq r_{2 n+1}
$$

From (10) and (12) we obtain

$$
r_{n+1} \leq r_{n}
$$

From (8) \& (11) for all $n=1,2,3, \cdots$, we have

$$
\int_{0}^{\phi_{1}\left(r_{n+1}\right)} \eta(y) \mathrm{d} y \leq \int_{0}^{\psi_{1}\left(r_{n}\right)} \eta(y) \mathrm{d} y-\int_{0}^{\psi_{2}\left(r_{n}\right)} \eta(y) \mathrm{d} y
$$

then

$$
\int_{0}^{\phi_{2}\left(r_{n+1}\right)} \eta(y) \mathrm{d} y \leq \int_{0}^{\phi_{1}\left(r_{n}\right)} \eta(y) \mathrm{d} y-\int_{0}^{\phi_{1}\left(r_{n+1}\right)} \eta(y) \mathrm{d} y
$$


Taking summation in above equation we obtain

$$
\sum_{0}^{\infty} \int_{0}^{\phi_{2}\left(r_{n+1}\right)} \eta(y) \mathrm{d} y \leq \int_{0}^{\phi_{1}\left(r_{0}\right)} \eta(y) \mathrm{d} y<\infty
$$

which implies $\phi_{2}(r) \rightarrow 0$ as $n \rightarrow \infty$.

Now from (13) sequence $\left\{r_{n}\right\}$ is convergent and as $n \rightarrow \infty, r_{n} \rightarrow r$. We know that $\phi$ is continuous and from Equation (14) we obtain $\phi_{2}(r)=0$ which implies that $r=0$, i.e. as

$n \rightarrow \infty, \quad r=\sigma\left(e_{n+1}, e_{n}\right) \rightarrow 0$.

We now show that the sequence $\left\{e_{n}\right\}$ is a Cauchy sequence in E. Keeping in mind Equation (15) it is require to show that $\left\{e_{2 s}\right\}_{s=1}^{\infty} \subset\left\{e_{n}\right\}$ is a Cauchy sequence. If $\left\{e_{2 s}\right\}_{s=1}^{\infty}$ is not a Cauchy sequence of natural number $\{2 m(k)\},\{2 n(k)\}$ such that $n(k)>m(k), \sigma\left(e_{2 m(k)}, e_{2 n(k)}\right) \geq \in$

$$
\sigma\left(e_{2 m(k)}, e_{2 n(k)-1}\right)<\epsilon
$$

Hence using (16)

$$
\begin{aligned}
\in & <\sigma\left(e_{2 m(k)}, e_{2 n(k)}\right) \\
& \leq \sigma\left(e_{2 m(k)}, e_{2 n(k)-1}\right)+\sigma\left(e_{2 n(k)}, e_{2 n(k)-1}\right) \\
& <\in+\sigma\left(e_{2 n(k)}, e_{2 n(k)-1}\right) .
\end{aligned}
$$

Taking $k \rightarrow \infty$ in the inequality above \& by result of Equation (15), we arrive at

$$
\lim _{n \rightarrow \infty} \sigma\left(e_{2 m(k)}, e_{2 n(k)}\right)=\in
$$

For all $k=1,2,3, \cdots$

$$
\sigma\left(e_{2 n(k)+1}, e_{2 m(k)}\right) \leq \sigma\left(e_{2 n(k)+1}, e_{2 n(k)}\right)+\sigma\left(e_{2 n(k)}, e_{2 m(k)}\right)
$$

Also for $k=1,2,3, \cdots$

$$
\sigma\left(e_{2 n(k)}, e_{2 m(k)}\right) \leq \sigma\left(e_{2 n(k)}, e_{2 n(k)+1}\right)+\sigma\left(e_{2 n(k)+1}, e_{2 m(k)}\right) .
$$

Making $k \rightarrow \infty$ in (18) \& (19) respectively by using (15) \& (17) we have

$$
\begin{gathered}
\lim _{k \rightarrow \infty} \sigma\left(e_{2 n(k)+1}, e_{2 m(k)}\right) \leq \epsilon \\
\text { and } \in \leq \lim _{k \rightarrow \infty} \sigma\left(e_{2 n(k)+1}, e_{2 m(k)}\right)
\end{gathered}
$$

Therefore, $\lim _{k \rightarrow \infty} \sigma\left(e_{2 n(k)+1}, e_{2 m(k)}\right)=\in$, for $k=1,2,3, \cdots$

$$
\begin{aligned}
& \sigma\left(e_{2 n(k)}, e_{2 m(k)-1}\right) \leq \sigma\left(e_{2 n(k)}, e_{2 m(k)}\right)+\sigma\left(e_{2 m(k)}, e_{2 m(k)-1}\right), \\
& \sigma\left(e_{2 n(k)}, e_{2 m(k)}\right) \leq \sigma\left(e_{2 n(k)}, e_{2 m(k)-1}\right)+\sigma\left(e_{2 m(k)-1}, e_{2 m(k)}\right) .
\end{aligned}
$$

Taking $k \rightarrow \infty$ in the above two inequalities and using (15) \& (17) we obtain

$$
\lim _{n \rightarrow \infty} \sigma\left(e_{2 n(k)}, e_{2 m(k)-1}\right)=\in \text {. }
$$




$$
\begin{gathered}
\text { Putting } c=e_{2 n(k)}, d=e_{2 m(k)-1} \text { in (8), for all } k=1,2,3, \cdots, \text { we obtain } \\
\int_{0}^{\phi_{1} \sigma\left(e_{2 n(k)+1}, e_{2 m(k)}\right)} \eta(y) \mathrm{d} y=\int_{0}^{\phi_{1} \sigma\left(U e_{2 n(k)}, V e_{2 m(k)-1}\right)} \eta(y) \mathrm{d} y \\
\leq \int_{0}^{\psi_{1}\left(\sigma\left(e_{2 n}, e_{2 m(k)-1}\right), \sigma\left(e_{2 n(k)+1}, e_{2 n}\right), \sigma\left(e_{2 m(k)}, e_{2 m(k)-1}\right), \frac{1}{2}\left\{\sigma\left(e_{2 m(k)}, e_{2 n(k)}\right)+\sigma\left(e_{2 n(k)+1}, e_{2 m(k)-1}\right)\right\}, \frac{1}{2}\left\{\sigma\left(e_{2 n(k)}, e_{2 m(k)-1}\right)+\sigma\left(e_{2 n(k)+1}, e_{2 n}(k)\right)\right\}\right)} \eta(y) \mathrm{d} y \\
-\int_{0}^{\psi_{2}\left(\sigma\left(e_{2 n}, e_{2 m(k)-1}\right), \sigma\left(e_{2 n(k)+1}, e_{2 n}\right), \sigma\left(e_{2 m(k)}, e_{2 m(k)-1}\right), \frac{1}{2}\left\{\sigma\left(e_{2 m(k)}, e_{2 n(k)}\right)+\sigma\left(e_{2 n(k)+1}, e_{2 m(k)-1}\right)\right\}, \frac{1}{2}\left\{\sigma\left(e_{2 n(k)}, e_{2 m(k)-1}\right)+\sigma\left(e_{2 n(k)+1}, e_{2 n}(k)\right)\right\}\right)} \eta(y) \mathrm{d} y
\end{gathered}
$$

Now in above inequality if we take $k \rightarrow \infty$ and by using results of (15), (20) $\&(21)$ we get

$$
\int_{0}^{\phi_{1}(\epsilon)} \eta(y) \mathrm{d} y \leq \int_{0}^{\psi_{1}\left(\epsilon, 0,0, \epsilon, \frac{1}{2} \epsilon\right)} \eta(y) \mathrm{d} y-\int_{0}^{\psi_{2}\left(\epsilon, 0,0, \in, \frac{1}{2} \epsilon\right)} \eta(y) \mathrm{d} y .
$$

Then $\phi_{1}(\in) \leq \psi_{1}\left(\in, 0,0, \in, \frac{1}{2} \in\right)-\psi_{2}\left(\in, 0,0, \in, \frac{1}{2} \in\right)=\phi_{1}(\in)$.

This is due to monotone increasing fact of $\psi_{1}$ in its variable and by using property of $\psi_{2}$ that $\psi_{2}\left(y_{1}, y_{2}, y_{3}, y_{4}, y_{5}\right)=0$, if and only if $y_{1}=y_{2}=y_{3}=y_{4}=y_{5}=0$.

From the above inequality we get a contradiction. So that $\in=0$. This establishes convergent sequence in $(E, \sigma)$.

Let $e_{n} \rightarrow z$ as $n \rightarrow \infty$.

Substituting $c=e_{2 n}, \quad d=z$ in (8) for all $n=1,2,3, \cdots$

$$
\begin{aligned}
& \int_{0}^{\phi_{1}\left(\sigma\left(e_{2 n+1}, V z\right)\right)} \eta(y) \mathrm{d} y \\
& \leq \int_{0}^{\psi_{1}\left(\sigma\left(e_{2 n}, z\right), \sigma\left(e_{2 n+1}, e_{2 n}\right), \sigma(V z, z), \frac{1}{2}\left\{\sigma\left(V z, e_{2 n}\right)+\sigma\left(e_{2 n+1}, z\right)\right\},, \frac{1}{2}\left\{\sigma\left(e_{2 n}, z\right)+\sigma\left(e_{2 n+1}, e_{2 n}\right)\right\}\right)} \eta(y) \mathrm{d} y \\
& -\int_{0}^{\psi_{2}\left(\sigma\left(e_{2 n}, z\right), \sigma\left(e_{2 n+1}, e_{2 n}\right), \sigma(V z, z), \frac{1}{2}\left\{\sigma\left(V z, e_{2 n}\right)+\sigma\left(e_{2 n+1}, z\right)\right\}, \frac{1}{2}\left\{\sigma\left(e_{2 n}, z\right)+\sigma\left(e_{2 n+1}, e_{2 n}\right)\right\}\right)} \eta(y) \mathrm{d} y
\end{aligned}
$$

Taking limit $\mathrm{n}$ tends to infinity in the above inequality and using continuity of $\psi_{1}$ and $\psi_{2}$ and Equations (15), (22) we get

$$
\begin{aligned}
& \int_{0}^{\phi_{1}(\sigma(z, V z))} \eta(y) \mathrm{d} y \\
& \leq \int_{0}^{\psi_{1}\left(\sigma(z, z), \sigma(z, z), \sigma(V z, z), \frac{1}{2}\{\sigma(V z, z)\}, 0\right)} \eta(y) \mathrm{d} y-\int_{0}^{\psi_{2}\left(\sigma(z, z), \sigma(z, z), \sigma(V z, z), \frac{1}{2}\{\sigma(V z, z)\}, 0\right)} \eta(y) \mathrm{d} y \\
& \leq \int_{0}^{\psi_{1}\left(0,0, \sigma(V z, z), \frac{1}{2}\{\sigma(V z, z)\}, 0\right)} \eta(y) \mathrm{d} y-\int_{0}^{\psi_{2}\left(0,0, \sigma(V z, z), \frac{1}{2}\{\sigma(V z, z)\}, 0\right)} \eta(y) \mathrm{d} y
\end{aligned}
$$

If $(V z, z) \neq 0$ then monotone increasing $\psi_{1}$ and $\psi_{2}$ are monotone increasing and $\psi_{2}\left(y_{1}, y_{2}, y_{3}, y_{4}, y_{5}\right)=0$, if and only if $y_{1}=y_{2}=y_{3}=y_{4}=y_{5}=0$, we obtain

$$
\int_{0}^{\phi_{1}(\sigma(z, V z)} \eta(y) \mathrm{d} y \leq \int_{0}^{\phi_{1}(\sigma(z, V z)} \eta(y) \mathrm{d} y
$$

This contradiction, hence we obtain $(V z, z)=0$.

In similar way we prove that $z=U z$. Hence $z=U z=V z$.

Hence (25) \& (26) shows that $z$ is a common fixed point of $U$ and $V$.

Theorem 2.2: Let $(E, \sigma)$ be a complete metric space and $p, q, U$ and $V$ be four mappings from $E$ to itself such that 


$$
\begin{aligned}
& \int_{0}^{\phi_{1}(\sigma(p s, q t))} \eta(y) \mathrm{d} y \\
& \leq \int_{0}^{\left.\psi_{1}\left(\sigma(U s, V t), \sigma(U s, q t), \sigma(p s, V t), \sigma(U s, p s), \sigma(V t, q t), \frac{1}{2}\{\sigma(q t, U s)+\sigma(p s, V t)\}\right), \frac{1}{2}\{\sigma(U s, V t)+\sigma(p s, U s)\}\right)} \eta(y) \mathrm{d} y \\
& \quad-\int_{0}^{\psi_{2}\left(\sigma(U s, V t), \sigma(U s, q t), \sigma(p s, V t), \sigma(U s, p s), \sigma(V t, q t), \frac{1}{2}\{\sigma(q t, U S)+\sigma(p s, V t)\}, \frac{1}{2}[\sigma(U s, V t)+\sigma(p s, U S))\right\}} \eta(y) \mathrm{d} y,
\end{aligned}
$$

for all $s, t \in E$, where $\psi_{1}, \psi_{2} \in \Psi_{7}, \phi_{1}=\psi(e, e, e, e, e, e, e)$, for $e \in[0, \infty)$.

i: One of the four mappings $p, q, U$ and $V$ is continuous.

ii: $(p, U) \&(q, V)$ are sub compatible.

iii: The pairs $p(s) \subseteq V(s)$ and $q(s) \subseteq U(s)$.

iv: Where $\eta: R^{+} \rightarrow R^{+}$is Lebesgue-integrable mappings, which is sum able, non negative and such that for each $\in>0, \int_{0}^{\epsilon} \eta(y) d y>0$.

Then $p, q, U$ and $V$ have a unique common fixed point in $E$.

Proof: Consider arbitrary point $e_{0} \in E$, we construct the sequence $\left\{e_{n}\right\}$ and $\left\{w_{n}\right\}$ in $E$ such that

$$
p e_{2 n}=V e_{2 n+1}=w_{2 n} \text { and } q e_{2 n+1}=U e_{2 n+2}=w_{n+1}, \quad n=0,1,2, \cdots
$$

Let $r_{n}=\sigma\left(w_{n}, w_{n+1}\right)$, Substitution $s=e_{2 n}$ and $t=e_{2 n+1}$ in (27) we have

$$
\begin{aligned}
& \int_{0}^{\phi_{1}\left(\sigma\left(p e_{2 n}, q e_{2 n+1}\right)\right)} \eta(y) \mathrm{d} y=\int_{0}^{\phi_{1}\left(\sigma\left(e_{2 n+1}, e_{2 n+2}\right)\right)} \eta(y) \mathrm{d} y \\
& \psi_{1}\left(\sigma\left(U e_{2 n}, V e_{2 n+1}\right), \sigma\left(U e_{2 n}, q e_{2 n+1}\right), \sigma\left(p e_{2 n+1}, V e_{2 n+1}\right), \sigma\left(U e_{2 n}, p e_{2 n}\right), \sigma\left(V e_{2 n+1}, q e_{2 n+1}\right),\right. \\
& \left.\leq \int_{0} \frac{1}{2}\left\{\sigma\left(q e_{2 n+1}, U e_{2 n}\right)+\sigma\left(p e_{2 n}, V e_{2 n+1}\right)\right\}, \frac{1}{2}\left\{\sigma\left(U e_{2 n}, V e_{2 n+1}\right)+\sigma\left(p e_{2 n}, U e_{2 n}\right)\right\}\right) \quad \eta(y) \mathrm{d} y \\
& \psi_{2}\left(\sigma\left(U e_{2 n}, V e_{2 n+1}\right), \sigma\left(U e_{2 n}, q e_{2 n+1}\right), \sigma\left(p e_{2 n+1}, V e_{2 n+1}\right), \sigma\left(U e_{2 n}, p e_{2 n}\right), \sigma\left(V e_{2 n+1}, q e_{2 n+1}\right),\right. \\
& \left.-\int_{0} \frac{1}{2}\left\{\sigma\left(q e_{2 n+1}, U e_{2 n}\right)+\sigma\left(p e_{2 n}, V e_{2 n+1}\right)\right\}, \frac{1}{2}\left\{\sigma\left(U e_{2 n}, V e_{2 n+1}\right)+\sigma\left(p e_{2 n}, U e_{2 n}\right)\right\}\right) \quad \eta(y) \mathrm{d} y \\
& \int_{0}^{\phi\left(\sigma\left(w_{2 n}, w_{2 n+1}\right)\right)} \eta(y) \mathrm{d} y \\
& \psi_{1}\left(\sigma\left(w_{2 n-1}, w_{2 n}\right), \sigma\left(w_{2 n-1}, w_{2 n+1}\right), \sigma\left(w_{2 n+1}, w_{2 n}\right), \sigma\left(w_{2 n-1}, w_{2 n}\right), \sigma\left(w_{2 n}, w_{2 n+1}\right),\right. \\
& \left.\leq \int_{0} \frac{1}{2}\left\{\sigma\left(w_{2 n+1}, w_{2 n}\right)+\sigma\left(w_{2 n}, w_{2 n}\right)\right\}, \frac{1}{2} \sigma\left(w_{2 n-1}, w_{2 n}\right)+\sigma\left(w_{2 n}, w_{2 n-1}\right)\right) \quad \eta(y) \mathrm{d} y \\
& \psi_{2}\left(\sigma\left(w_{2 n-1}, w_{2 n}\right), \sigma\left(w_{2 n-1}, w_{2 n+1}\right), \sigma\left(w_{2 n+1}, w_{2 n}\right), \sigma\left(w_{2 n-1}, w_{2 n}\right), \sigma\left(w_{2 n}, w_{2 n+1}\right),\right. \\
& \left.-\int_{0} \frac{1}{2}\left\{\sigma\left(w_{2 n+1}, w_{2 n}\right)+\sigma\left(w_{2 n}, w_{2 n}\right)\right\}, \frac{1}{2} \sigma\left(w_{2 n-1}, w_{2 n}\right)+\sigma\left(w_{2 n}, w_{2 n-1}\right)\right) \quad \eta(y) d y \\
& \int_{0}^{\phi_{1}\left(r_{2 n}\right)} \eta(y) \mathrm{d} y \leq \int_{0}^{\psi_{1}\left(r_{2 n-1}, r_{2 n-1}+r_{2 n}, r_{2 n}, r_{2 n-1}, r_{2 n}, \frac{1}{2}\left\{r_{2 n}\right\}, \frac{1}{2}\left\{r_{2 n-1}+r_{2 n-1}\right\}\right)} \eta(y) \mathrm{d} y \\
& -\int_{0}^{\psi_{2}\left(r_{2 n-1}, r_{2 n-1}+r_{2 n}, r_{2 n}, r_{2 n-1}, r_{2 n}, \frac{1}{2}\left\{r_{2 n}\right\}, \frac{1}{2}\left\{r_{2 n-1}+r_{2 n-1}\right\}\right)} \eta(y) \mathrm{d} y
\end{aligned}
$$

If $r_{2 n+1} \leq r_{2 n}$ then $r_{2 n+1}+r_{2 n} \leq 2 r_{2 n}$ and

$$
\begin{aligned}
\int_{0}^{\phi_{1}\left(r_{2 n}\right)} \eta(y) \mathrm{d} y \leq & \int_{0}^{\psi_{1}\left(r_{2 n-1}, 2 r_{2 n}, r_{2 n}, r_{2 n-1}, r_{2 n}, \frac{1}{2}\left\{r_{2 n}\right\}, r_{2 n-1}\right)} \eta(y) \mathrm{d} y \\
& -\int_{0}^{\psi_{2}\left(r_{2 n-1}, 2 r_{2 n}, r_{2 n}, r_{2 n-1}, r_{2 n}, \frac{1}{2}\left\{r_{2 n}\right\}, r_{2 n-1}\right)} \eta(y) \mathrm{d} y \\
< & \int_{0}^{\phi_{1}\left(r_{2 n}\right)} \eta(y) \mathrm{d} y .
\end{aligned}
$$

Thus we arrive at a contradiction. Hence $r_{2 n} \leq r_{2 n-1}$, similarly by substituting $s=r_{2 n+2}, t=r_{2 n+1}$ in (27) we can prove that, $r_{2 n+1} \leq r_{2 n}$, for $n=0,1,2, \cdots$. Thus $r_{n+1} \leq r_{n}$, for $n=0,1,2, \cdots$. Hence the sequence $\left\{r_{n}\right\}$ is sequence of positive real numbers, which is decreasing and converges to $r \in R$.

Let $m=\lim _{n \rightarrow \infty} \frac{1}{2} d\left(w_{n}, w_{n+2}\right)$. Taking $n \rightarrow \infty$ in (27) we have 


$$
\begin{gathered}
\int_{0}^{\phi_{1}\left(r_{2 n}\right)} \eta(y) \mathrm{d} y \leq \int_{0}^{\psi_{1}(r, r, r, r, r, r, r)} \eta(y) \mathrm{d} y-\int_{0}^{\psi_{2}(r, r, r, r, r, r, m)} \eta(y) \mathrm{d} y \\
\quad \leq \int_{0}^{\phi_{1}(r)} \eta(y) \mathrm{d} y-\int_{0}^{\psi_{2}(r, r, r, r, r, r, m)} \eta(y) \mathrm{d} y . \\
\text { Thus } \psi_{2}(r, r, r, r, r, r, m)=0 \text { So that } r=m=0 . \\
\text { Hence } \lim _{n \rightarrow \infty} d\left(y_{n}, y_{n+1}\right)=0
\end{gathered}
$$

In view of (29), to prove sequence $\left\{w_{n}\right\}$ is a Cauchy sequence it is sufficient to prove the subsequence $\left\{w_{2 n}\right\}$ of sequence $\left\{w_{n}\right\}$ is a Cauchy sequence. If $\left\{w_{2 n}\right\}$ is not a Cauchy sequence there exist $\in>0$ \& sequence of natural numbers $\{2 m(k)\} \&\{2 n(k)\}$ which are monotone increasing such that $n(k)>m(k)$.

$$
\sigma\left(w_{2 m(k)}, w_{2 n(k)}\right) \geq \in \& \sigma\left(w_{2 m(k)}, w_{2 n(k)-2}\right)<\epsilon .
$$

Then from (29) we have

$$
\begin{aligned}
\in & <\sigma\left(w_{2 m(k)}, w_{2 n(k)}\right) \\
& \leq \sigma\left(w_{2 m(k)}, w_{2 n(k)-2}\right)+\sigma\left(w_{2 n(k)-1}, w_{2 n(k)-2}\right)+\sigma\left(w_{2 n(k)-1}, w_{2 n(k)}\right) \\
& <\in+\sigma\left(w_{2 n(k)-1}, w_{2 n(k)-2}\right)+\sigma\left(w_{2 n(k)-1}, w_{2 n(k)}\right) .
\end{aligned}
$$

Taking $k \rightarrow \infty$ and using (29) we have

$$
\lim _{n \rightarrow \infty} \sigma\left(w_{2 m(k)}, w_{2 n(k)}\right)=\in \text {. }
$$

Taking $k \rightarrow \infty$ using (29) \& (30) in

$$
\left|\sigma\left(w_{2 m(k)}, w_{2 n(k)+1}\right)-\sigma\left(w_{2 m(k)}, w_{2 n(k)}\right)\right| \leq \sigma\left(w_{2 n(k)}, w_{2 n(k)+1}\right) .
$$

We get $\lim _{n \rightarrow \infty} \sigma\left(w_{2 n(k)+1}, w_{2 m(k)}\right)=\epsilon$.

Letting $k \rightarrow \infty$ and from Equations (29) \& (30) in

$$
\left|\sigma\left(w_{2 m(k)-1}, w_{2 n(k)}\right)-\sigma\left(w_{2 m(k)}, w_{2 n(k)}\right)\right| \leq \sigma\left(w_{2 m(k)}, w_{2 m(k)-1}\right) .
$$

We get $\lim _{k \rightarrow \infty} \sigma\left(w_{2 m(k)}, w_{2 m(k)-1}\right)=\epsilon$.

Putting $s=x_{2 m(k)}, t=x_{2 n(k)-1}$ in (27), for all $k=1,2,3, \cdots \quad$ we obtain

$$
\begin{aligned}
& \int_{0}^{\phi_{1}\left(p x_{2 m(k)}, q x_{2 n(k)-1}\right)} \eta(y) \mathrm{d} y \\
& \psi_{1}\left(\sigma\left(U x_{2 m(k)}, V X_{2 m(k)-1}\right), \sigma\left(U x_{2 m(k)}, q x_{2 n(k)-1}\right), \sigma\left(p x_{2 m(k)}, V X_{2 n(k)-1}\right), \sigma\left(U x_{2 m(k)}, p x_{2 m(k)}\right), \sigma\left(V x_{2 n(k)-1}, q x_{2 n(k)-1}\right)\right. \\
& \left.\leq \int_{0} \frac{1}{2}\left\{\sigma\left(q x_{2 n(k)-1}, U x_{2 m(k)}\right)+\sigma\left(p x_{2 m(k)}, V X_{2 n(k)-1}\right)\right\}, \frac{1}{2}\left\{\sigma\left(U x_{2 m(k)}, V X_{2 n(k)-1}\right)+\sigma\left(p x_{2 m(k)}, U x_{2 m(k)}\right)\right\}\right) \quad \eta(y) \mathrm{d} y \\
& \psi_{2}\left(\sigma\left(U x_{2 m(k)}, V x_{2 m(k)-1}\right), \sigma\left(U x_{2 m(k)}, q x_{2 n(k)-1}\right), \sigma\left(p x_{2 m(k)}, V x_{2 n(k)-1}\right), \sigma\left(U x_{2 m(k)}, p x_{2 m}(k), \sigma\left(V x_{2 n}(k)-1, q x_{2 n}(k)-1\right)\right.\right. \\
& \left.-\int_{0} \frac{1}{2}\left\{\sigma\left(q x_{2 n(k)-1}, U x_{2 m(k)}\right)+\sigma\left(p x_{2 m(k)}, V x_{2 n(k)-1}\right)\right\}, \frac{1}{2}\left\{\sigma\left(U x_{2 m(k)}, V x_{2 n(k)-1}\right)+\sigma\left(p x_{2 m(k)}, U x_{2 m(k)}\right)\right\}\right) \\
& -\int_{0} \\
& \psi_{1}\left(\sigma\left(w_{2 m(k)-1}, w_{2 n(k)-2}\right), \sigma\left(w_{2 m(k)-1}, w_{2 n(k)-1}\right), \sigma\left(w_{2 m(k)}, w_{2 n(k)-2}\right), \sigma\left(w_{2 m(k)-1}, w_{2 m(k)}\right), \sigma\left(w_{2 m(k)-2}, w_{2 n(k)-1}\right),\right. \\
& \leq \int_{0} \\
& \left.\frac{1}{2}\left\{\sigma\left(w_{2 n(k)-1}, w_{2 m(k)-1}\right)+\sigma\left(w_{2 m(k)}, w_{2 n(k)-2}\right)\right\}, \frac{1}{2}\left\{\sigma\left(w_{2 m(k)-1}, w_{2 n(k)-2}\right)+\sigma\left(w_{2 m(k)}, w_{2 n(k)-1}\right)\right\}\right) \\
& \psi_{2}\left(\sigma\left(w_{2 m(k)-1}, w_{2 n(k)-2}\right), \sigma\left(w_{2 m(k)-1}, w_{2 n(k)-1}\right), \sigma\left(w_{2 m(k)}, w_{2 n(k)-2}\right), \sigma\left(w_{2 m(k)-1}, w_{2 m(k)}\right), \sigma\left(w_{2 m(k)-2}, w_{2 n(k)-1}\right),\right. \\
& \left.\frac{1}{2}\left\{\sigma\left(w_{2 n(k)-1}, w_{2 m(k)-1}\right)+\sigma\left(w_{2 m(k)}, w_{2 n(k)-2}\right)\right\}, \frac{1}{2}\left\{\sigma\left(w_{2 m(k)-1}, w_{2 n(k)-2}\right)+\sigma\left(w_{2 m(k)}, w_{2 n(k)-1}\right)\right\}\right) \\
& -\int_{0} \\
& \eta(y) \mathrm{d} y \\
& \eta(y) \mathrm{d} y \\
& \eta(y) \mathrm{d} y
\end{aligned}
$$


Taking $k \rightarrow \infty$ \& using (29), (30), (32), (33) \& (35) we get

$$
\begin{aligned}
\int_{0}^{\phi_{1}(\epsilon)} \eta(y) \mathrm{d} y & \leq \int_{0}^{\psi_{1}\left(\epsilon, \epsilon, \in, 0,0, \frac{1}{2}[\epsilon+\epsilon], \frac{1}{2} \epsilon\right)} \eta(y) \mathrm{d} y-\int_{0}^{\psi_{2}\left(\epsilon, \epsilon, \epsilon, 0,0, \frac{1}{2}[\epsilon+\epsilon], \frac{1}{2} \epsilon\right)} \eta(y) \mathrm{d} y \\
& <\int_{0}^{\psi_{1}(\epsilon \in \in \in \in \in, \epsilon, \epsilon, \epsilon)} \eta(y) \mathrm{d} y=\int_{0}^{\phi_{1}(\epsilon)} \eta(y) \mathrm{d} y .
\end{aligned}
$$

This is contradiction. Hence $\left\{w_{2 n}\right\}$ is a Cauchy sequence and is convergent. Since $E$ is complete there exist $z \in E$ such that as $n \rightarrow \infty$ we have $w_{n} \rightarrow z$.

Case I: Assume that $U$ is continuous then $U p e_{2 n} \rightarrow U z, U^{2} e_{2 n} \rightarrow U z$. Since $(p, U)$ is sub compatible, we have $p U e_{2 n} \rightarrow U z$.

Step I: Substituting $s=U e_{2 n}, t=e_{2 n+1}$ in (27), we have

$$
\begin{aligned}
& \int_{0}^{\phi_{1} \sigma\left(p U e_{2 n}, q e_{2 n+1}\right)} \eta(y) \mathrm{d} y \\
& \psi_{1}\left(\sigma\left(U^{2} e_{2 n}, V e_{2 n+1}\right), \sigma\left(U^{2} e_{2 n}, q e_{2 n+1}\right), \sigma\left(p U e_{2 n}, V e_{2 n+1}\right), \sigma\left(U^{2} e_{2 n}, p U e_{2 n}\right), \sigma\left(V e_{2 n+1}, q e_{2 n+1}\right),\right. \\
& \left.\leq \int_{0} \frac{1}{2}\left\{\sigma\left(q e_{2 n+1}, U^{2} e_{2 n}\right)+\sigma\left(p U e_{2 n}, V e_{2 n+1}\right)\right\}, \frac{1}{2}\left\{\sigma\left(U^{2} e_{2 n}, V e_{2 n+1}\right)+\sigma\left(p U e_{2 n}, U^{2} e_{2 n}\right)\right\}\right) \quad \eta(y) \mathrm{d} y \\
& \psi_{2}\left(\sigma\left(U^{2} e_{2 n}, V e_{2 n+1}\right), \sigma\left(U^{2} e_{2 n}, q e_{2 n+1}\right), \sigma\left(p U e_{2 n}, V e_{2 n+1}\right), \sigma\left(U^{2} e_{2 n}, p U e_{2 n}\right), \sigma\left(V e_{2 n+1}, q e_{2 n+1}\right),\right. \\
& \left.-\int_{0} \frac{1}{2}\left\{\sigma\left(q e_{2 n+1}, U^{2} e_{2 n}\right)+\sigma\left(p U e_{2 n}, V e_{2 n+1}\right)\right\}, \frac{1}{2}\left\{\sigma\left(U^{2} e_{2 n}, V e_{2 n+1}\right)+\sigma\left(p U e_{2 n}, U^{2} e_{2 n}\right)\right\}\right) \quad \eta(y) \mathrm{d} y, \\
& \int_{0}^{\phi_{1}(\sigma(U z, z))} \eta(y) \mathrm{d} y \\
& \leq \int_{0}^{\psi_{1}\left(\sigma(U z, z), \sigma(U z, z), \sigma(U z, z), \sigma(U z, U z), \sigma(z, z), \frac{1}{2}\{\sigma(z, U z)+\sigma(U z, z)\}, \frac{1}{2}\{\sigma(U z, z)+\sigma(U z, U z)\}\right)} \eta(y) \mathrm{d} y \\
& -\int_{0}^{\psi_{2}\left(\sigma(U z, z), \sigma(U z, z), \sigma(U z, z), \sigma(U z, U z), \sigma(z, z), \frac{1}{2}\{\sigma(z, U z)+\sigma(U z, z)\}, \frac{1}{2}\{\sigma(U z, z)+\sigma(U z, U z)\}\right)} \eta(y) \mathrm{d} y \\
& \leq \int_{0}^{\psi_{1}\left(\sigma(U z, z), \sigma(U z, z), \sigma(U z, z), 0,0, \sigma(U z, z), \frac{1}{2}\{\sigma(U z, z)\}\right)} \eta(y) \mathrm{d} y \\
& \leq \int_{0}^{\phi_{1}(\sigma(U z, z))} \eta(y) \mathrm{d} y .
\end{aligned}
$$

It is contradiction if $U z \neq z$. Hence $U z=z$.

Step II: Substituting $s=z, t=e_{2 n+1}$ in (27) and taking limit as $n$ tends to infinity we get $p z=z$.

Step III: We know that $z=p z \in p(e) \subseteq V(e)$ then there exist $u \in E$ such that $z=V u$. Substituting $s=e_{2 n}, t=u$ in (27) we get $q z=z$. Hence $q z=z=V z$ and $q V u=V q u$, which gives $q z=V z$.

Step IV: Substituting $s=z, t=z$ in (27) we have $q z=z$ so that $q(z)=z=V z$. Hence $p, q, U \& V$ have a common fixed point $z$ in $E$.

Case II: Assume that $U$ is continuous then $p^{2} e_{2 n} \rightarrow p z, \quad p U e_{2 n} \rightarrow p z$. Similarly we can prove that $z$ is common fixed point of $p, q, U \& V$. When $q$ or $V$ is continuous, then the uniqueness of common fixed point follows easily from (27).

Example: Let $E=[0,1]$ with the usual metric $\sigma(s, t)=\frac{1}{2}|s-t|$. Define $p, q, U, V: E \rightarrow E$ such that $p s=\frac{s}{4}, q t=\frac{t}{4}, U s=s, V t=t$.

Let $\psi_{1}\left(y_{1}, y_{2}, y_{3}, y_{4}, y_{5}, y_{6}, y_{7}\right)=\max \left(y_{1}, y_{2}, y_{3}, y_{4}, y_{5}, y_{6}, y_{7}\right), \varphi(y)=2 y$, $\psi_{2}=\frac{1}{4} \psi_{1}$ then $\psi_{1}(y)=y, \forall y \in[0, \infty)$ 


$$
\begin{aligned}
\left|\frac{s}{4}-\frac{t}{4}\right|^{2} \leq \frac{1}{4} \max & \left\{\sigma(s, t), \sigma\left(s, \frac{t}{4}\right), \sigma\left(\frac{s}{4}, t\right), \sigma\left(s, \frac{s}{4}\right), \sigma\left(t, \frac{t}{4}\right),\right. \\
& \left.\frac{1}{2}\left\{\sigma\left(\frac{t}{4}, s\right)+\sigma\left(\frac{s}{4}, t\right)\right\}, \frac{1}{2}\left\{\sigma(s, t)+\sigma\left(\frac{s}{4}, s\right)\right\}\right\}
\end{aligned}
$$

For all $s, t \in E$, it follows that the condition (27).

Let $\left\{e_{n}\right\}$ be a sequence in $E$ such that $p e_{n} \rightarrow z \& U e_{n} \rightarrow z$ for some $z$ in $E$. Then $z=0, \sigma\left(p U e_{n}, U p e_{n}\right) \rightarrow 0$. Hence $\{p, U\}$ is sub compatible. We have common fixed point in $\mathrm{E}$.

\section{Conclusion}

In this paper, we proved the fixed point theorem for four sub compatible maps under a contractive condition of integral type. These results can be extended to any directions and can also be extended to fixed point theory of non-expansive multi-valued mappings.

\section{Acknowledgements}

The authors would like to give their sincere thanks to the editor and the anonymous referees for their valuable comments and useful suggestions in improving the article.

\section{References}

[1] Khan, M.S., Swaleh, M. and Sessa, S. (1984) Fixed Point Theorems by Altering Distances between Two Points. Bulletin of the Australian Mathematical Society, 30, 1 9.

[2] Aliouche (2006) A Common Fixed Point Theorem for Weakly Compatible Mappings in Symmetric Spaces Satisfying a Contractive Condition of Integral Type. Journal of Mathematical Analysis and Applications, 322, 796-802.

[3] Babu, G.V.R. (2004) Generalization of Fixed Point Theorems Relating to the Diameter of Orbits by Using a Control Function. Tamkang Journal of Mathematics, 63, 159-168.

[4] Bouhadjera, H. and Godet, C. (2009) The Common Fixed Point Theorem for a Pair of Sub Compatible Maps. http://hal.archives-ouvertes.fr/hal-00356516v2

[5] Choudhury, B.S. (2005) A Common Unique Fixed Point Result in Metric Spaces Involving Generalized Altering Distances. Mathematical Communications, 14, 105110.

[6] Choudhury, B.S. and Dutta, P.N. (2000) A Unified Fixed Point Result in Metric Spaces Involving a Two Variable Function. Filomat, 14, 43-48.

[7] Chugh, R. and Kumar, S. (2001) Common Fixed Points for Weakly Compatible Maps. Proceedings of the Indian Academy of Sciences-Mathematical Sciences, 111, 241-247.

[8] Naidu, S.V.R. (2001) Fixed Point Theorem by Altering Distances. Adv. Math. Sci. Appl., 11, 1-6.

[9] Sastry, K.P.R., Naidu, S.V.R., Babu, G.V.R. and Naidu, G.A. (2000) The Generalization of Common Fixed Point Theorems for Weakly Commuting Maps by Altering Distances. Tamkang Journal of Mathematics, 31, 243-250. 
[10] Hosseni, V.R. and Hossseni, N. (2012) Common Fixed Point Theorems for Maps Altering Distance under Contractive Condition of Integral Type for Pairs of Sub Compatible Maps. International Journal of Math Analysis, 6, 1123-1130.

[11] Hosseni, V.R. and Hossseni, N. (2010) Common Fixed Point Theorems by Altering Distance Involving under Contractive Condition of Integral Type. International Mathematical Forum, 5, 1951-1957.

[12] Wadkar, B.R., Bhardwaj, R.K. and Singh, B. (2014) A Common Fixed Point Theorem in Dislocated Metric Space. International Journal of Engineering Research and Development, 10, 14-17.

[13] Mishra, L.N., Mishra, V.N., Khatri, K. and Deepmala (2014) On the Trigonometric Approximation of Signals Belonging to Generalized Weighted Lipschitz $W\left(L^{r}\right.$, $\xi(t))(r \geq 1)$-class by matrix $\left(C \cdot N_{p}\right)$ Operator of Conjugate Series of Its Fourier Series. Applied Mathematics and Computation, 237, 252-263.

https://doi.org/10.1016/j.amc.2014.03.085

[14] Mishra, V.N., Khatri, K. and Mishra, L.N. (2013) Using Linear Operator to Approximate Signal of $\operatorname{Lip}(\alpha, p),(p \geq 1)$-Class. Filomat, 27, 355-365.

https://doi.org/10.2298/FIL1302353M

[15] Mishra, L.N., Tiwari, S.K., Mishra, V.N. and Khan, I.A. (2015) Unique Fixed Point Theorems for Generalized Contractive Mappings in Partial Metric Spaces. Journal of Function Spaces, 2015, Article ID: 960-827.

[16] Mishra, L.N., Tiwari, S.K. and Mishra, V.N. (2015) Fixed Point Theorems for Generalized Weakly S-Contractive Mappings in Partial Metric Spaces. Journal of Applied Analysis and Computation, 5, 600-612.

[17] Deepmala and Pathak, H.K. (2013) A Study on Some Problems on Existence of Solutions for Nonlinear Functional-Integral Equations. Acta Mathematica Scientia, 33, 1305-1313. https://doi.org/10.1016/S0252-9602(13)60083-1

[18] Wadkar, B.R., Bhardwaj, R. and Singh, B. (2017) Fixed Point Theorems in Dislocated Metric Space. Global Journal of Pure and Applied Mathematics, 13, 20892110.

[19] Wadkar, B.R., Bhardwaj, R., Mishra, V.N. and Singh, B. (2017) Coupled Fixed Point Theorems in Soft Metric and b-Soft Metric Space. Ser. A: Appl. Math. Inform. and Mech., 9, 59-73.

[20] Wadkar, B.R., Bhardwaj, R., Singh, B. and Mishra, V.N. (2017) Coupled Fixed Point Theorems with Monotone Property in Soft Metric and Soft b-Metric Space. International Journal of Mathematical Analysis, 11, 363-375. http://dx.doi.org/10.12988/7222

[21] Wadkar, B.R., Bhardwaj, R., Mishra, V.N. and Singh, B. (2016) Fixed Point Results Related to Soft Sets. Australian Journal of Basic and Applied Sciences, 10, 128-137. 
Submit or recommend next manuscript to SCIRP and we will provide best service for you:

Accepting pre-submission inquiries through Email, Facebook, LinkedIn, Twitter, etc. A wide selection of journals (inclusive of 9 subjects, more than 200 journals)

Providing 24-hour high-quality service

User-friendly online submission system

Fair and swift peer-review system

Efficient typesetting and proofreading procedure

Display of the result of downloads and visits, as well as the number of cited articles Maximum dissemination of your research work

Submit your manuscript at: http://papersubmission.scirp.org/

Or contact apm@scirp.org 